\title{
DIFFUSION IN NOVAE AT HIGH ACCRETION RATES
}

\author{
Attay Kovetz, School of Physics and Astronomy, and \\ Dina Prialnik, Department of Geophysics and Planetary Sciences \\ Raymond and Beverly Sackler Faculty of Exact Sciences \\ Tel Aviv University, Ramat Aviv 69978, Israel
}

\section{Background}

The main outstanding problem in our full understanding of the classical nova mechanism is the apparent discrepancy between mass transfer rates $\left(10^{-9}\right.$ to $\left.10^{-8} M_{\odot} / \mathrm{yr}\right)$ inferred from observations (Patterson, 1984, Ap.J.Suppl. 231, 789), and those required by numerical models in order to reproduce nova characteristics $\left(10^{-11}\right.$ to $\left.10^{-9} M_{\odot} / \mathrm{yr}\right)$. The low accretion rates are needed in order to obtain powerful runaways and high values of $\mathrm{Z}$ in the ejecta by the diffusion-convection mechanism. The discrepancy seems to have sharpened by the realization that accretional heating (Shaviv and Starrfield, 1987, Ap.J. 321, L51) and angular momentum transfer (Sparks and Kutter, 1987, Ap.J. 321, 394 and Kutter and Sparks, 1987, Ap.J. 321, 386) tend to lower the theoretical upper limit for $\dot{M}$ to about $10^{-10} M_{\odot} /$ yr. On the other hand, scenarios invoking variable mass accretion rates - hibernation (Shara et al, 1986, Ap.J. 311, 163) or 'mild' hibernation (Livio, Shankar and Truran, 1988, Ap.J. 330, 264) - have shown that the high observed rates immediately prior and following outbursts can be reconciled with lower average accretion rates over the period between outbursts. Hence accretion rates of order $10^{-10} M_{\odot} / \mathrm{yr}$ may resolve the controversy between theory and observations, if they can be shown to lead to strong nova outbursts with high-Z ejecta. This prompted us to carry out fresh calculations of the accretion-diffusion-runaway-convection process for $\dot{M}=10^{-10}$ and $10^{-9} M_{\odot} / \mathrm{yr}$, taking into account accretional heating and using an improved diffusion algorithm.

\section{Assumptions}

Our initial model was a white dwarf with the following characteristics: $M_{W D}=$ $1.25 M_{\odot}, L_{W D}=0.01 L_{\odot}, T_{W D}=15.8710^{6} \mathrm{~K}, X_{C}=0.50, X_{O}=0.50$. Fine zoning (down to $\Delta m=10^{-8} M_{\odot}$ ) was used in the outer layers.

Material with $X=.735, Y=.249$ and solar heavy-element composition was accreted at the two following rates: $\dot{M}=10^{-9} M_{\odot} /$ yr and $\dot{M}=10^{-10} M_{\odot} /$ yr. Accretional heating at the stellar surface was taken to be $0.2 L_{a c c}=0.2 \frac{G M \dot{M}}{R}$, in accordance with the recent estimate of Regev (1988, Proc. I.A.U. Coll. No. 114, p. 519). The models rapidly adjusted to this heating, and their luminosities during the accretion phase were $L=1.6 L_{\odot}$ and $L=0.16 L_{\odot}$.

Diffusion (of all isotopes) was calculated in accordance with the scheme of Prialnik and Kovetz (1984, Ap.J. 281, 367). 


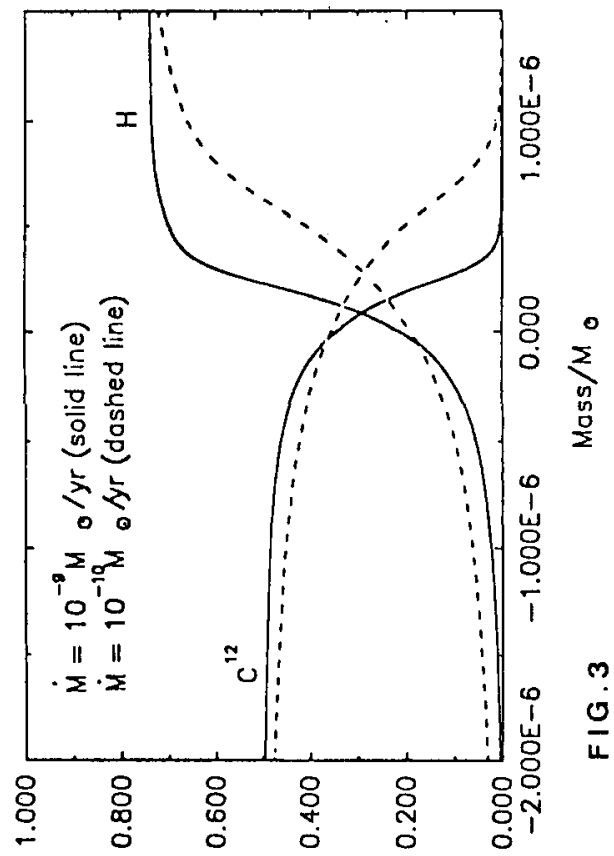

UO!PODIf SSDW

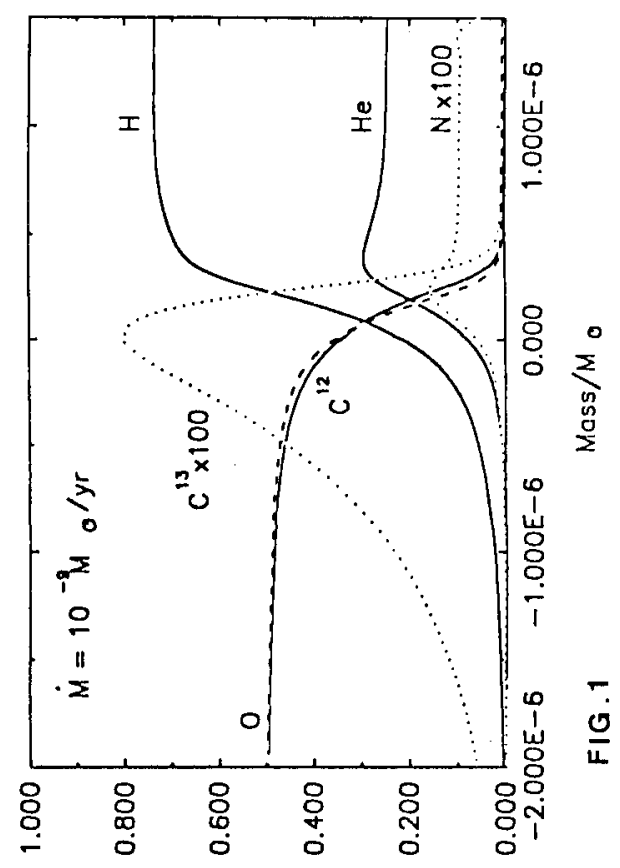

UO!fODAf ssow

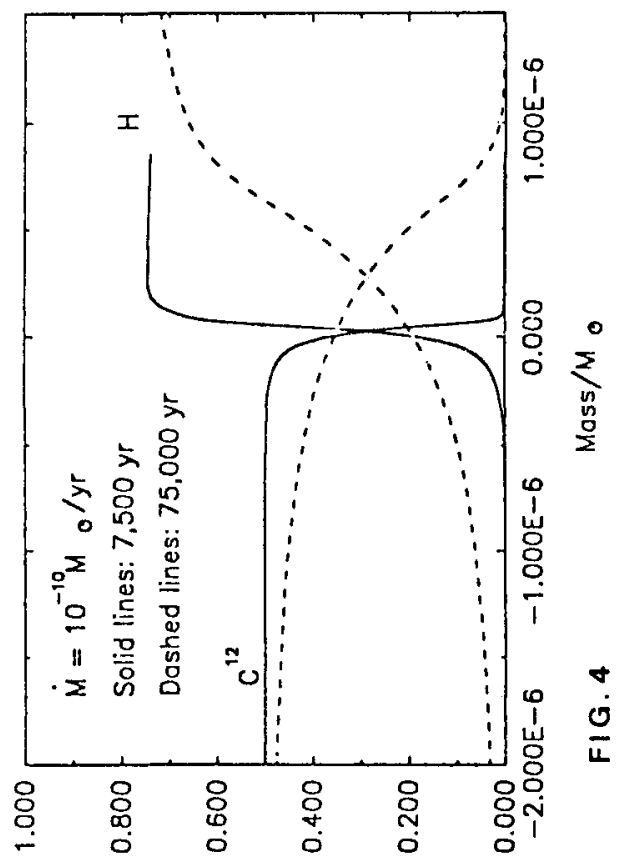

uo!foDdf ssow

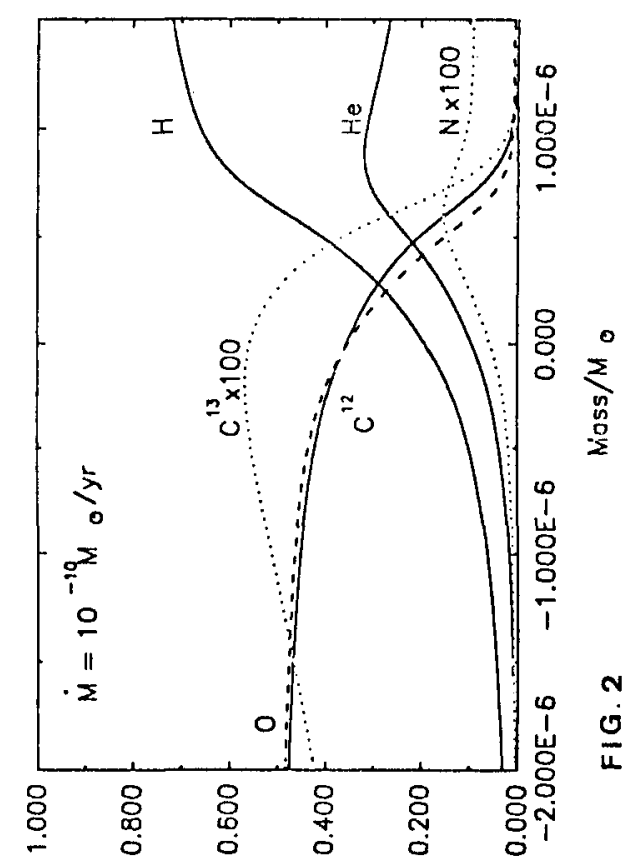

uo!foDy ssow 


\section{Results}

The first four figures show composition profiles resulting from diffusion. The mass abscissa in these figures has its zero point at the original position of the interface between the $\mathrm{C}-\mathrm{O}$ core and the accreted, hydrogen-rich envelope. In Figures 1 and 2 the profiles for the two models are shown just prior to the onset of convection at the beginning of the thermonuclear runaway. At this stage the age of the $10^{-9} M_{\odot} / \mathrm{yr}$ model is 7,500 yr, about ten times less than that of the slowly accreting $10^{-10} M_{\odot} / \mathrm{yr}$ model.

It would be wrong to conclude that diffusive composition changes in an accreting white dwarf proceed linearly with time and are therefore inversly proportional to $\dot{M}$. This is because diffusion is mainly driven by (partial) pressure forces, which increase with depth. Figures 3 and 4, in which only hydrogen and carbon profiles are shown, demonstrate this point. Figure 3 is a superposition of $\mathrm{H}$ and $\mathrm{C}$ profiles from Figures 1 and 2, while in Figure 4 the profiles for the $10^{-9} M_{\odot} / \mathrm{yr}$ model are replaced by those of the $10^{-10} M_{\odot} / \mathrm{yr}$ model at $t=7,500 \mathrm{yr}$. In spite of the fact that the solid lines in Figures 3 and 4 correspond to the same age, the effect of diffusion is considerably stronger in the case of the higher accretion rate.

The next figure is a compilation of the final heavy-element mass fraction $\mathrm{Z}$ in nova ejecta obtained from calculations which were all based on diffusive mixing. The legend for this figure is the following: open triangles - Kovetz and Prialnik (1985, Ap.J. 291, 812); open squares - Prialnik and Shara (1986, Ap.J. 311, 172); inverted filled triangle - Prialnik (1986, Ap.J. 310, 222); filled diamonds - this calculation. The figure shows clearly that the final $\mathrm{Z}$ is not uniquely determined by $\dot{M}$. It also depends on other parameters, such as the mass and the luminosity of the accreting white dwarf, the accretion history (in the case of a variable $\dot{M}$ ) and the composition of the outer white dwarf layers.

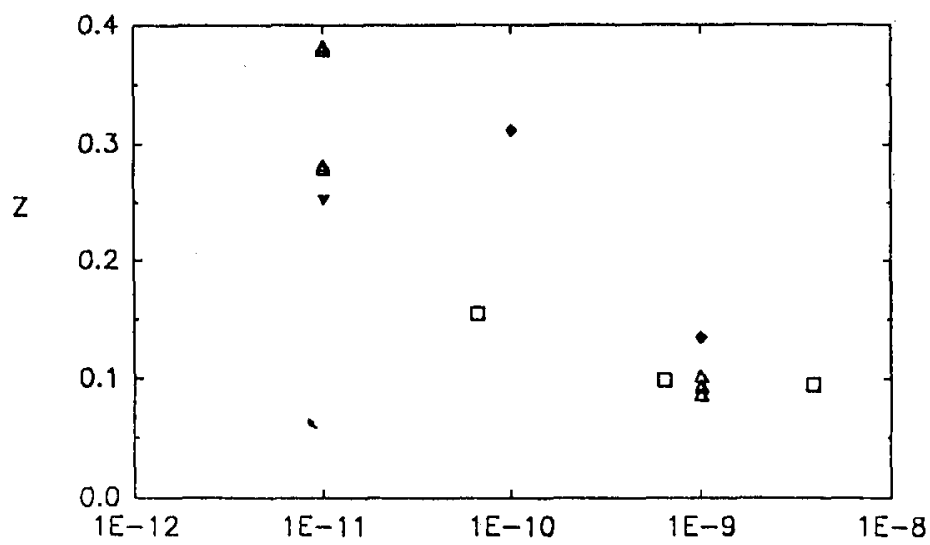

Accretion rate $(M \circ / y r)$

FIGURE 5 